\title{
Absorption and impedance boundary conditions for phased geometrical-acoustics methods
}

Jeong, Cheol-Ho

Published in:

Acoustical Society of America. Journal

Link to article, DOI:

$10.1121 / 1.4740494$

Publication date:

2012

Link back to DTU Orbit

Citation (APA):

Jeong, C-H. (2012). Absorption and impedance boundary conditions for phased geometrical-acoustics methods. Acoustical Society of America. Journal, 132(4), 2347-2358. https://doi.org/10.1121/1.4740494

\section{General rights}

Copyright and moral rights for the publications made accessible in the public portal are retained by the authors and/or other copyright owners and it is a condition of accessing publications that users recognise and abide by the legal requirements associated with these rights.

- Users may download and print one copy of any publication from the public portal for the purpose of private study or research.

- You may not further distribute the material or use it for any profit-making activity or commercial gain

- You may freely distribute the URL identifying the publication in the public portal

If you believe that this document breaches copyright please contact us providing details, and we will remove access to the work immediately and investigate your claim 


\title{
Absorption and impedance boundary conditions for phased geometrical-acoustics methods
}

\author{
Cheol-Ho Jeong ${ }^{\text {a) }}$ \\ Acoustic Technology, Department of Electrical Engineering, Technical University of Denmark, DK-2800 \\ Kongens Lyngby, Denmark
}

(Received 2 May 2012; revised 29 June 2012; accepted 6 July 2012)

\begin{abstract}
Defining accurate acoustical boundary conditions is of crucial importance for room acoustic simulations. In predicting sound fields using phased geometrical acoustics methods, both absorption coefficients and surface impedances of the boundary surfaces can be used, but no guideline has been developed on which boundary condition produces accurate results. In this study, various boundary conditions in terms of normal, random, and field incidence absorption coefficients and normal incidence surface impedance are used in a phased beam tracing model, and the simulated results are validated with boundary element solutions. Two rectangular rooms with uniform and non-uniform absorption distributions are tested. Effects of the neglect of reflection phase shift are also investigated. It is concluded that the impedance, random incidence, and field incidence absorption boundary conditions produce reasonable results with some exceptions at low frequencies for acoustically soft materials. (C) 2012 Acoustical Society of America. [http://dx.doi.org/10.1121/1.4740494]
\end{abstract}

PACS number(s): 43.55.Ka, 43.55.Ev [NX]

Pages: $2347-2358$

\section{INTRODUCTION}

The acoustic properties of building elements such as sound absorption coefficients or surface impedances are crucial input data for room acoustic simulations. Absorption coefficients are mainly used for geometrical acoustics methods, whereas wave-based methods generally require surface impedance data. Phase geometrical acoustics methods can adopt both absorption coefficient and surface impedance boundary conditions. This paper primarily aims to evaluate various approximate boundary conditions for a phased beam tracing model. The main question is which boundary condition for the phased geometrical acoustics best approximates locally reacting boundaries. As a validation tool, the boundary element method employing the identical surface impedance boundary condition is used.

The phased geometrical acoustics methods have obvious advantages over the other methods at medium frequencies as they are faster than wave-based methods and more accurate than energy-based methods. ${ }^{1-3}$ Generally speaking, wave based methods are the most reliable methods in calculating transfer functions at low frequencies, whereas the geometrical acoustics methods provide approximate but reliable and fast predictions at frequencies well above the Schroeder frequency where room modes are highly overlapped; thus individual modal characters do not need to be taken into account. In this sense, the phased geometrical acoustics methods are normally considered as medium frequency methods that can bridge between low and high frequency methods. A recent publication supports that the accuracy of a phased geometrical acoustics turns out to be acceptable even below the Schroder frequency, having a maximum

\footnotetext{
a) Author to whom correspondence should be addressed. Electronic mail: chj@elektro.dtu.dk
}

error of $3 \mathrm{~dB}$ for a disproportionate room. ${ }^{3}$ Therefore the phased geometrical acoustics methods can be alternatives to the wave-based methods in the acoustic simulations of small spaces such as car cabins, small class rooms, and meeting rooms as long as there is no significant effect of diffracting objects.

In addition, its calculation speed is faster than the wave-based method. For example, a narrow band transfer function calculation from $20 \mathrm{~Hz}$ to $1 \mathrm{kHz}$ at $2 \mathrm{~Hz}$ intervals of a $1000 \mathrm{~m}^{3}$ room, employing 8000 beams up to the 100 th specular reflection order, takes $1.5 \mathrm{~h}$, while a boundary element calculation takes $56.5 \mathrm{~h}^{3}$ The advantage in terms of the calculation cost becomes significant as the room becomes larger and the upper frequency limit gets higher. An important advantage over the geometrical acoustics methods is flexible boundary modeling. The conventional geometrical acoustics methods can employ only absorption coefficient boundary conditions, whereas the phased geometrical acoustics methods can adopt both absorption and impedance data as boundary conditions. Furthermore, an advanced transfer-matrix approach for multilayer boundary surfaces can be incorporated to a phased beam tracing method. $^{4}$

Surface impedance boundary conditions are likely to be superior to absorption boundary conditions because they fully describe the physical characteristics of the boundary, i.e., the magnitude and phase changes on reflections. It has been reported that phased geometrical acoustics simulations using surface impedances as boundary conditions agree well with measurements and reference calculations. ${ }^{1-3}$ Suh and Nelson used surface impedance boundary conditions for important surfaces to estimate the plane wave reflection coefficients, whereas real-valued angle-independent reflection coefficients are used for concrete and plaster walls, assuming no phase shift on reflections from these reflective surfaces. ${ }^{1}$ Lam mainly studied the plane and spherical wave reflection 
coefficients in a phased image source model in comparisons with boundary element simulations and geometrical acoustic simulations. ${ }^{2}$ The plane wave reflection model was found to have noticeable errors at higher admittance values and at longer delay time, but the accuracy was improved as the frequency increased. Jeong and Ih also used impedance boundary conditions for a phased beam tracing model in comparison with boundary element simulations and acoustic Green's function calculations. ${ }^{3}$

The normal incidence specific surface impedance $\left(\zeta_{\text {nor }}\right)$ is a typical boundary condition in the phased beam tracing as well as the boundary element simulation. The use of $\zeta_{\text {nor }}$ implies that the absorber is of local reaction because the surface impedance is assumed to be constant over the angle of incidence. The normal incidence surface impedance is generally complex-valued and frequency-dependent but often approximated to real-valued, therefore the reflection coefficient is also real-valued. Another impedance boundary condition is the field incidence surface impedance used by Aretz and Vorländer, ${ }^{5}$ which assumes nonlocally reacting surfaces. Therefore this quantity is omitted because only local reaction is assumed in this study.

If measured impedance data are not available, one has to approximately estimate absorption coefficients of the boundary surfaces. There are a variety of absorption coefficients to be used in the phased beam tracing: normal incidence, random incidence, field incidence, and Sabine absorption coefficients, which are all assumed to be independent of the incidence angle. However, no comparisons have been conducted between absorption and impedance boundary conditions, and this is one of the important purposes of this study.

A variety of approximate absorption coefficients can be used as boundary conditions in the geometrical acoustics models: normal, random, field incidence absorption coefficient $\left(\alpha_{\text {nor, }} \alpha_{\text {rand, }} \alpha_{\text {field }}\right)$, and Sabine absorption coefficient $\left(\alpha_{\mathrm{Sab}}\right){ }^{6}{ }^{6} \alpha_{\text {nor }}$ holds only for normal incidence. $\alpha_{\text {rand }}$ strictly assumes random incidence of randomly phased plane waves onto a large panel, which is unlikely in actual sound fields. $\alpha_{\text {field }}$ is an empirical modification of the random incidence absorption coefficient by truncating the integration range up to $78^{\circ} .^{6}$ The inclusion of $\alpha_{\text {field }}$ is based on a recent study by Aretz and Vorländer, claiming that the field incidence surface impedance produces the best results in finite element modeling. ${ }^{5} \alpha_{\text {Sab }}$ is measured in a reverberant sound field based on the assumption that the test chamber is completely diffuse. ${ }^{6,7}$ The calculation of $\alpha_{\text {Sab }}$ additionally assumes that the total absorption area is a simple sum of the absorption areas of individual surfaces. The total absorption area is calculated by Sabine's formula that again assumes a totally diffuse field. These many assumptions lead to a physically impossible consequence that $\alpha_{\mathrm{Sab}}$ sometimes exceeds unity, thereby cannot be used in room acoustic simulations. Besides, $\alpha_{\mathrm{Sab}}$ has large room-to-room variations as significant deviations in measured metrics have been found in round robin tests. ${ }^{8,9}$ This also indicates that it is likely that the acoustic behavior of the tested absorber in different acoustic conditions can differ significantly from that in the test reverberation chamber.
Two rectangular rooms with various uniform boundary conditions and one non-uniform distribution were simulated. As boundary conditions, the surface impedance and corresponding absorption coefficient were used. Two error indicators were defined to quantify the discrepancies between boundary element solutions and phased beam tracing solutions. Furthermore, effects of reflection phase shift on the simulation accuracy were investigated by employing various complex-valued impedance boundary conditions. All in all, the goal of this study is to find out the boundary condition that best approximates a locally reacting boundary in rectangular rooms and how the simulation error changes with various boundary conditions for the phased beam tracing method.

\section{THEORY}

\section{A. Normal incidence specific surface impedance}

This quantity can be measured by the tube method, whcih can characterize the impedance at a certain surface for normal incidence of plane waves. The specific surface impedance is normalized by the characteristic impedance of air by Eq. (1) and will be simply called as the impedance in what follows.

$$
\zeta_{\text {nor }}=\frac{Z_{\text {nor }}}{\rho c} \text {. }
$$

\section{B. Normal incidence absorption coefficient}

The normal incidence absorption coefficient is also measured by the tube method in the following relationship to the impedance:

$$
\alpha_{\text {nor }}=1-\left|\frac{\zeta_{\text {nor }}-1}{\zeta_{\text {nor }}+1}\right|^{2}
$$

\section{Random incidence absorption coefficient}

The theoretical random incidence absorption coefficient for plane wave incidence on an infinitely large surface can be calculated as follows: ${ }^{10}$

$$
\alpha_{\mathrm{rand}}=\int_{0}^{\pi / 2} \alpha_{\mathrm{inf}}\left(\theta_{i}\right) \sin \left(2 \theta_{i}\right) d \theta_{i}
$$

where $\alpha_{\text {inf }}\left(\theta_{i}\right)$ is the oblique incidence absorption coefficient at an incidence angle $\theta_{i}$ as follows:

$$
\alpha_{\text {inf }}\left(\theta_{i}\right)=\frac{4 \operatorname{Re}\left(\zeta_{\text {nor }}\right) \cos \theta_{i}}{\left(\left|\zeta_{\text {nor }}\right| \cos \theta_{i}\right)^{2}+2 \operatorname{Re}\left(\zeta_{\text {nor }}\right) \cos \theta_{i}+1} .
$$

It assumes that the intensity of the incident sound is uniformly distributed over all possible directions, and the phases of the waves incident on the absorber are randomly distributed. The random incidence absorption coefficient should have a value less than unity.

\section{Field incidence absorption coefficient}

The field incidence absorption coefficient is, admittedly, not common, and its definition is still debatable. Following 
the notation by Aretz and Vorländer ${ }^{5}$ and Maekawa et al., ${ }^{6}$ the field incidence absorption coefficient is calculated by

$$
\alpha_{\text {field }}=\frac{\int_{0}^{78} \alpha_{\text {inf }}\left(\theta_{i}\right) \sin \left(\theta_{i}\right) d \theta_{i}}{\int_{0}^{78} \sin \left(\theta_{i}\right) d \theta_{i}} .
$$

\section{E. Sabine absorption coefficients}

The Sabine absorption coefficient is an estimated absorption coefficient by the reverberation chamber method and Sabine's formula as follows: ${ }^{7}$

$$
\alpha_{\mathrm{Sab}}=\frac{55.3 \mathrm{~V}}{S}\left(\frac{1}{c_{2} T_{2}}-\frac{1}{c_{1} T_{1}}\right),
$$

where $V$ is the volume of the reverberation chamber, $S$ is the specimen area, and $c_{1}$ and $T_{1}$ are the speed of sound and the reverberation time for an empty condition, respectively. $c_{2}$ and $T_{2}$ are the speed of sound and the reverberation time with the specimen installed, respectively.

The measurement standards recommend a large specimen, i.e., a surface area between 10 and $12 \mathrm{~m}^{2}$ according to ISO 354 (Ref. 7) and larger than around $5.6 \mathrm{~m}^{2}$ according to ASTM C423. ${ }^{11}$ Even for large specimens, the Sabine absorption coefficients depend heavily on the test facilities, because the test chambers are non-diffuse in significantly different ways. Significant deviations in measured metrics have been found in many round robin tests (see, e.g., Refs. 8 and 9), therefore the universal use of Sabine absorption coefficients measured in a specific chamber is questionable. In this sense, this quantity will not be tested in this study.

\section{F. Remarks on local reaction}

This study assumes local reaction, which means that the wave transmitted into a porous material is refracted so that it propagates effectively only perpendicular to the surface. ${ }^{12}$ This is likely to happen in anisotropic solids such as honeycomb structures where the waves are forced to propagate perpendicular to the surface. Local reaction is also related to the ratio of the incident wave speed to the transmitted wave speed. Local reaction occurs, when the speed of the compressional wave in air is much higher than that of waves in an anisotropic solid, $c_{t} \ll c_{i}$, where the subscripts $i$ and $t$ denote the incidence and transmitted, respectively. By Snell's law, the angle of transmission, $\theta_{t}$, becomes much less than the incidence angle, $\theta_{i}$, resulting in a marked bending of the transmitted wave toward the normal direction. Therefore it is reasonable to assume that the ratio of acoustic pressure acting on the surface to the normal fluid velocity is independent of the incidence angle.

The assumption of local reaction has been widely used in room acoustic simulations using both wave-based and geometrical acoustics methods. The main reason is its simplicity compared with non-local reaction models, because knowledge of the normal surface impedance suffices for calculating the absorption characteristic of the wall, whereas extended reaction models necessitate, at least, a characteristic impedance and propagation constant.

Note, however, that local reaction is a simplification in the boundary modeling. No materials are strictly of local reaction; the surface impedance actually changes with the incidence angle. In certain cases, the assumption of local reaction is not adequate, e.g., porous layers backed by an air cavity because the refracted wave in the air cavity will propagate in the same direction as the initial incidence angle according to Snell's law. Several authors have measured extended reaction in porous materials. ${ }^{13-16}$ The local and non-local reaction models are known to agree better for higher resistivity cases and thicker absorbers. Detailed guidelines can be found in Ref. 17. Hodgson and Wareing also found large differences in predicted sound pressure levels based on local and non-local reaction at low frequencies. $^{18}$

\section{METHOD}

\section{A. Test rooms}

Narrow band spectra at $2 \mathrm{~Hz}$ intervals were calculated for two rooms that are different in shape and volume: a well proportionate room with dimensions of $1.9 \times 1.4 \times 1$ (all in $\mathrm{m}$ throughout the article) and a disproportionate room of dimensions of $5 \times 1 \times 1$. The first room ratio is based on Louden's work, which concluded that this room ratio is optimum for achieving evenly spaced modes. ${ }^{19}$ For the proportionate room, one source and 54 receivers were chosen. The source position was $(0.1,0.1,0.4)$ representing a teacher because this room was regarded as a $1 / 4$ scale model of a lecture room. A total of 54 receivers were positioned with $x$ changing from 0.15 to 1.75 with steps of 0.2 and $y$ changing from 0.2 to 1.2 with steps of 0.2 and a fixed $z$ of 0.3 . For the disproportionate room, one source and 36 receivers were positioned. The source was located at $(0.1$, $0.1,0.4)$ and 36 receivers were positioned with $x$ changing from 0.5 to 4.5 with steps of 0.5 and $y$ changing from 0.2 to 0.8 with steps of 0.2 and a fixed $z$ of 0.3 .

\section{B. Boundary conditions}

Various uniform distributions of absorption in the test rooms were examined. A set of impedance values of 40, 20, $10,7,4$, with acronyms of $\mathrm{BC} 1-\mathrm{BC} 5$, was tested as shown in Table I, where the corresponding absorption coefficients are also shown, ranging from 0.1 to 0.8 . A realistic nonuniform distribution in the proportionate room was also tested. The ceiling, floor, and the side walls were assigned with impedance data of 5.9, 18, 38, which corresponded to random incidence absorption coefficients of $0.66,0.32$, and 0.17 , respectively. In addition, complex-valued boundary conditions were compared with real-valued boundary conditions to estimate how much the errors were increased by using approximate real-valued boundary conditions. The complex-valued boundary conditions were given in two ways. First, for a fixed magnitude of the impedance of 20, the phase angle of the impedance was changed to $30^{\circ}, 45^{\circ}$, or $60^{\circ}$. Second, for a fixed random incidence absorption 
TABLE I. Errors for five uniform absorption conditions and one non-uniform condition in the proportionate room. The surface impedance, corresponding normal, random, field incidence coefficients, and Schroeder frequencies are indicated. The lowest errors are indicated in bold.

\begin{tabular}{|c|c|c|c|c|c|c|c|c|c|c|c|c|c|}
\hline & \multirow[b]{2}{*}{$\zeta_{\text {nor }}$} & \multirow[b]{2}{*}{$\alpha_{\text {nor }}$} & \multirow[b]{2}{*}{$\alpha_{\text {rand }}$} & \multirow[b]{2}{*}{$\alpha_{\text {field }}$} & \multirow[b]{2}{*}{$f_{\mathrm{Sch}}$} & \multicolumn{4}{|c|}{ Mean $e_{1}$} & \multicolumn{4}{|c|}{ Mean $e_{2}$} \\
\hline & & & & & & $\alpha_{\text {nor }}$ & $\alpha_{\text {rand }}$ & $\alpha_{\text {field }}$ & $\zeta_{\text {nor }}$ & $\alpha_{\text {nor }}$ & $\alpha_{\text {rand }}$ & $\alpha_{\text {field }}$ & $\zeta_{\text {nor }}$ \\
\hline BC1 & 40 & 0.10 & 0.17 & 0.18 & 566 & 4.0 & 2.2 & 2.1 & 1.8 & 2.2 & 0.6 & 0.6 & 0.9 \\
\hline $\mathrm{BC} 2$ & 20 & 0.18 & 0.30 & 0.32 & 423 & 3.7 & 1.4 & 1.4 & 1.2 & 2.4 & 0.6 & 0.6 & 0.9 \\
\hline BC3 & 10 & 0.33 & 0.49 & 0.52 & 331 & 3.7 & 1.2 & 1.1 & 1.0 & 2.5 & 0.9 & 0.9 & 1.0 \\
\hline BC4 & 7 & 0.43 & 0.60 & 0.65 & 299 & 3.7 & 1.4 & 1.3 & 1.0 & 2.6 & 1.1 & 1.2 & 1.1 \\
\hline BC5 & 4 & 0.64 & 0.79 & 0.83 & 260 & 3.5 & 1.8 & 1.7 & 1.2 & 2.6 & 1.7 & 1.9 & 1.7 \\
\hline Non-uniform & & 0.21 & 0.31 & 0.33 & 414 & 3.5 & 2.5 & 2.5 & 2.2 & 2.9 & 1.2 & 1.2 & 1.5 \\
\hline
\end{tabular}

coefficient of 0.3 , three equivalent impedance values having the phase angles of $30^{\circ}, 45^{\circ}$, and $60^{\circ}$ were employed as boundary conditions and compared with the real-valued boundary condition. These complex-valued boundary conditions are shown in Tables II and III.

\section{Phased beam tracing method}

The basic idea of the phased geometrical acoustics is to retain phase information to account for wave phenomena, particularly interference. Inclusion of phase is twofold: phase shift on reflection and propagation phase. The former phase requires complex-valued boundary conditions such as surface impedances. The latter phase is simply implemented based on the traveling distance from the source to receiver $\left(r_{\text {travel }}\right)$ and taking into account a term, $\exp \left(-j k r_{\text {travel }}\right)$, in calculation of sound pressures, where $j$ is the imaginary unit, and $k$ is the wavenumber.

PBTM used in this study is based on the triangular beam tracing algorithm by Lewers ${ }^{20}$ but extended to include phase. The tracing algorithm consists of source generation, surfacegeometry definition, traces of beams, and receiver detection. Source division is based on an icosahedron, which makes the beam cross section an equilateral triangle. Then all edges of the equilateral triangles of the icosahedron are divided into $p$ equal lengths, resulting in a polygon with $20 \cdot p^{2}$ triangular faces. For each point receiver, the source is rotated so that a triangle faces toward the receiver to not miss the direct sound. Room boundary surfaces should be planar, which are mathematically modeled as $A_{i} x+B_{i} y+C_{i} z+D_{i}=0$. A trajectory of a beam is scanned by a combined process of determining the nearest plane, finding the new image source, and calculating the reflected vector. A beam is defined by a central axis and three boundary planes, each plane forming a side of the beam. Beams do not fragment on reflection, and the direction after reflection is determined entirely by its central axis. Once the trajectory of the beam is identified, the possibility that a point receiver is surrounded by the beam boundary planes is tested using the normal vectors of the boundary walls. Following a positive receiver point test, the complex pressure for the beam undergoing the reflection path is calculated, and finally the transfer function is constructed. Only specular reflections are counted.

Consequently, a PBTM result is a summation of the contributions of the emitted beams from a source, which hit a receiver in a room. Assuming a simple source emitting spherical waves, the free-space Green's function, ${ }^{21} A \cdot \exp (-j k r) / r$, is a basis to calculate the transfer function at an observation point where $r$ is the distance from the source to the observation point and $A$ is an arbitrary constant. For all simulations in this study, $A$ is assumed to be unity, therefore producing $1 \mathrm{~Pa}$ at $1 \mathrm{~m}$ from the source.

For each reflection, a reflection coefficient is multiplied to the free-space Green's function. For the impedance boundary conditions, the plane wave reflection coefficient is calculated as follows:

$$
r\left(\theta_{i}\right)=\frac{\zeta_{\text {nor }} \cos \left(\theta_{i}\right)-1}{\zeta_{\text {nor }} \cos \left(\theta_{i}\right)+1} .
$$

Therefore the reflection coefficient is a function of the incidence angle although the impedance is not angle dependent. Note that this coefficient is correct for large panels. For grazing incidence or relatively small sized panels, another reflection modeling could be used. ${ }^{22,23}$

For absorption coefficient boundary conditions, the angle dependence and phase shift on reflection are neglected.

TABLE II. Errors for different phase angles with a fixed magnitude of the impedance of 20 in the proportionate room. The surface impedance, corresponding normal, random, fiend incidence coefficients, and the Schroeder frequencies are indicated. $\zeta_{\text {nor, real }}$ indicates an error using the real-valued impedance boundary condition, and $\zeta_{\text {nor,correct }}$ indicates an error using the correct impedance boundary condition.

\begin{tabular}{|c|c|c|c|c|c|c|c|c|c|c|}
\hline & \multirow[b]{2}{*}{$\left|\zeta_{\text {nor }}\right|$} & \multirow[b]{2}{*}{$\mu$} & \multirow[b]{2}{*}{$\alpha_{\text {nor }}$} & \multirow[b]{2}{*}{$\alpha_{\text {rand }}$} & \multirow[b]{2}{*}{$\alpha_{\text {field }}$} & \multirow[b]{2}{*}{$f_{\text {Sch }}$} & \multicolumn{2}{|c|}{ Mean $e_{1}$} & \multicolumn{2}{|c|}{ Mean $e_{2}$} \\
\hline & & & & & & & $\zeta_{\text {nor,real }}$ & $\zeta_{\text {nor,correct }}$ & $\zeta_{\text {nor,real }}$ & $\zeta_{\text {nor,correct }}$ \\
\hline C-BC1 & 20 & 30 & 0.16 & 0.26 & 0.28 & 451 & 2.2 & 1.3 & 1.4 & 0.8 \\
\hline $\mathrm{C}-\mathrm{BC} 2$ & 20 & 45 & 0.13 & 0.20 & 0.23 & 492 & 3.2 & 1.6 & 2.3 & 1.0 \\
\hline C-BC3 & 20 & 60 & 0.10 & 0.15 & 0.17 & 572 & 4.4 & 2.0 & 3.5 & 1.2 \\
\hline BC2 & 20 & 0 & 0.18 & 0.30 & 0.32 & 423 & 1.2 & 1.2 & 0.9 & 0.9 \\
\hline
\end{tabular}


TABLE III. Errors for different phase angles with a fixed random incidence absorption coefficient of 0.3 in the proportionate room. $\alpha_{\text {rand }}$ indicates an error using the random incidence absorption boundary condition, and $\zeta_{\text {nor,correct }}$ indicates an error using the correct impedance boundary condition.

\begin{tabular}{|c|c|c|c|c|c|c|}
\hline & \multirow[b]{2}{*}{$\left|\zeta_{\text {nor }}\right|$} & \multirow[b]{2}{*}{$\mu$} & \multicolumn{2}{|c|}{ Mean $e_{1}$} & \multicolumn{2}{|c|}{ Mean $e_{2}$} \\
\hline & & & $\alpha_{\text {rand }}$ & $\zeta_{\text {nor,correct }}$ & $\alpha_{\text {rand }}$ & $\zeta_{\text {nor,correct }}$ \\
\hline $\mathrm{C} 2-\mathrm{BC} 1$ & 17.2 & 30 & 2.6 & 1.2 & 0.9 & 0.8 \\
\hline $\mathrm{C} 2-\mathrm{BC} 2$ & 13.8 & 45 & 3.5 & 1.4 & 1.5 & 1.0 \\
\hline C2-BC3 & 9.4 & 60 & 4.7 & 1.7 & 2.6 & 1.7 \\
\hline $\mathrm{BC} 2$ & 20 & 0 & 1.4 & 1.2 & 0.6 & 0.9 \\
\hline
\end{tabular}

Therefore a real-valued and positive reflection coefficient is calculated as

$$
r_{i}=\sqrt{1-\alpha_{i}},
$$

where the subscript $i$ can be "nor," "rand," or "field."

Challenges still remain in the phased beam tracing, particularly in considering diffraction and diffuse reflection. Another problem of PBTM arises when beams intersect more than one surface. If an intersecting polygon is detected, there are two solutions: The original beam is followed by its central axis ray ${ }^{4,20,22}$ or the original beam can be split. ${ }^{24,25}$ Beam-splitting algorithms possibly make simulations more accurate, but they are computationally voracious.

For all PBTM simulations, 8000 beams were emitted from the source, and they were traced up to the 100th reflection order. The reason for the large number of beams is due to the fact that PBTM used does not incorporate a beamsplitting algorithm when intersecting more than two surfaces. In addition, a sufficient number of late reflections should be calculated, therefore each beam was traced up to the 100 th reflection order. For BC1, the average propagation distance for the 100th reflection order is $97 \mathrm{~m}$, corresponding to a sufficient decay range of $45 \mathrm{~dB}$. For the other boundary conditions, the decay ranges are even longer as the reverberation times become shorter with increasing absorption.

\section{Boundary element models}

The boundary element method can solve acoustic problems numerically based on the discretized HelmholtzKirchhoff integral equation on a surface mesh. ${ }^{26}$ An inhouse boundary element model is used for comparisons. For the proportionate room, the boundary element model contains 6880 triangular elements with 3442 nodes of which the $6 \lambda$ per element condition is satisfied up to $1000 \mathrm{~Hz}$. For the disproportionate room, it has 7228 elements and 3616 nodes, therefore its upper cutoff frequency is about $700 \mathrm{~Hz}$. For the two rooms, the linear shape function and seven Gaussian points were used. The boundary element simulations are regarded as the reference simulations.

\section{E. Error measures}

In predicting transfer functions using both PBTM and $\mathrm{BEM}$, the source is assumed to produce a sound pressure of
$1 \mathrm{~Pa}$ at $1 \mathrm{~m}$ from the source in a free field. Once transfer functions at $2 \mathrm{~Hz}$ intervals are calculated using PBTM and $\mathrm{BEM}$, they are converted to the $\mathrm{dB}$ scale re. $1 \mathrm{~Pa}, S P L_{\mathrm{PBTM}}$ and $S P L_{\mathrm{BEM}}$, respectively. In addition, $1 / 3$ octave band spectra are computed based on the narrow band transfer functions, and named as $S P L_{\mathrm{PBTM}, o c t}$ and $S P L_{\mathrm{BEM}, \mathrm{oct}}$. Two errors are defined to compare phased beam tracing simulations with boundary element simulations: a narrow band error as $e_{1}$ by Eq. (9), and a $1 / 3$ octave band error as $e_{2}$ by Eq. (10)

$$
e_{1}\left(f_{c}\right)=\frac{1}{N_{\text {line }}} \sum_{i=f_{\text {low }}}^{f_{\text {up }}}\left|S P L_{\mathrm{PBTM}}(i)-S P L_{\mathrm{BEM}}(i)\right|(\mathrm{dB}),
$$

$$
e_{2}\left(f_{c}\right)=\left|S P L_{\mathrm{PBTM}, \mathrm{oct}}\left(f_{c}\right)-S P L_{\mathrm{BEM}, \mathrm{oct}}\left(f_{c}\right)\right|(\mathrm{dB}),
$$

where $f_{\text {up }}$ and $f_{\text {low }}$ are the upper and lower frequency limit of a frequency band, $f_{\mathrm{c}}$ is the center frequency of the band, and $N_{\text {line }}$ is the number of frequency lines in the frequency band of interest. The upper valid frequencies of the proportionate and disproportionate room boundary element models are 1000 and $700 \mathrm{~Hz}$, respectively, therefore the highest center frequencies of the $1 / 3$ octave band are $800 \mathrm{~Hz}$ for the proportionate room and $500 \mathrm{~Hz}$ for the disproportionate room. For single-value errors, these errors are averaged over the entire frequency range and over the receiver locations as shown in Tables I-IV.

Two main sources of error in PBTM have been known: errors at off-resonance frequencies due to geometrical tracing and sphericity error. ${ }^{2,3}$ The former error influences $e_{1}$ mainly for high impedances, whereas $e_{2}$, which is based on the sound pressure levels summed in $1 / 3$ octave bands, is seldom affected by the former error. However, the sphericity error arises at very low frequencies for low surface impedance. Because $e_{2}$ is the $1 / 3$ octave band error, which emphasizes low frequency errors more, the sphericity error can increase $e_{2}$ significantly. Therefore a larger $e_{2}$ than $e_{1}$ indicates that the sphericity error prevails, whereas the opposite holds for the error at off-resonance frequencies.

\section{RESULTS AND DISCUSSIONS}

\section{A. Uniform absorption in the proportionate room}

Average simulation errors in the proportionate room are shown in Table I and Figs. 1 and 2. In Table I, the frequency and receiver averaged errors are listed, where it is found that

TABLE IV. Errors for five uniform absorption conditions in the dispropor-

\begin{tabular}{|c|c|c|c|c|c|c|c|c|}
\hline & \multirow[b]{2}{*}{$\zeta_{\text {nor }}$} & \multirow[b]{2}{*}{$f_{\mathrm{Sch}}$} & \multicolumn{3}{|c|}{ Mean $e_{1}$} & \multicolumn{3}{|c|}{ Mean $e_{2}$} \\
\hline & & & $\alpha_{\text {nor }}$ & $\alpha_{\text {rand }}$ & $\zeta_{\text {nor }}$ & $\alpha_{\text {nor }}$ & $\alpha_{\text {rand }}$ & $\zeta_{\text {nor }}$ \\
\hline $\mathrm{BC} 1$ & 40 & 416 & 4.0 & 2.0 & 1.6 & 3.1 & 1.2 & 1.4 \\
\hline $\mathrm{BC} 2$ & 20 & 311 & 4.5 & 2.0 & 1.5 & 3.9 & 1.4 & 1.7 \\
\hline $\mathrm{BC} 3$ & 10 & 243 & 5.4 & 2.8 & 1.7 & 4.4 & 2.0 & 2.2 \\
\hline $\mathrm{BC} 4$ & 7 & 219 & 6.1 & 3.5 & 1.9 & 4.9 & 2.5 & 2.8 \\
\hline BC5 & 4 & 192 & 7.3 & 5.3 & 2.4 & 6.2 & 4.1 & 4.2 \\
\hline
\end{tabular}
tionate room. The surface impedance and corresponding Schroeder frequencies are shown. The lowest errors are indicated in bold. 

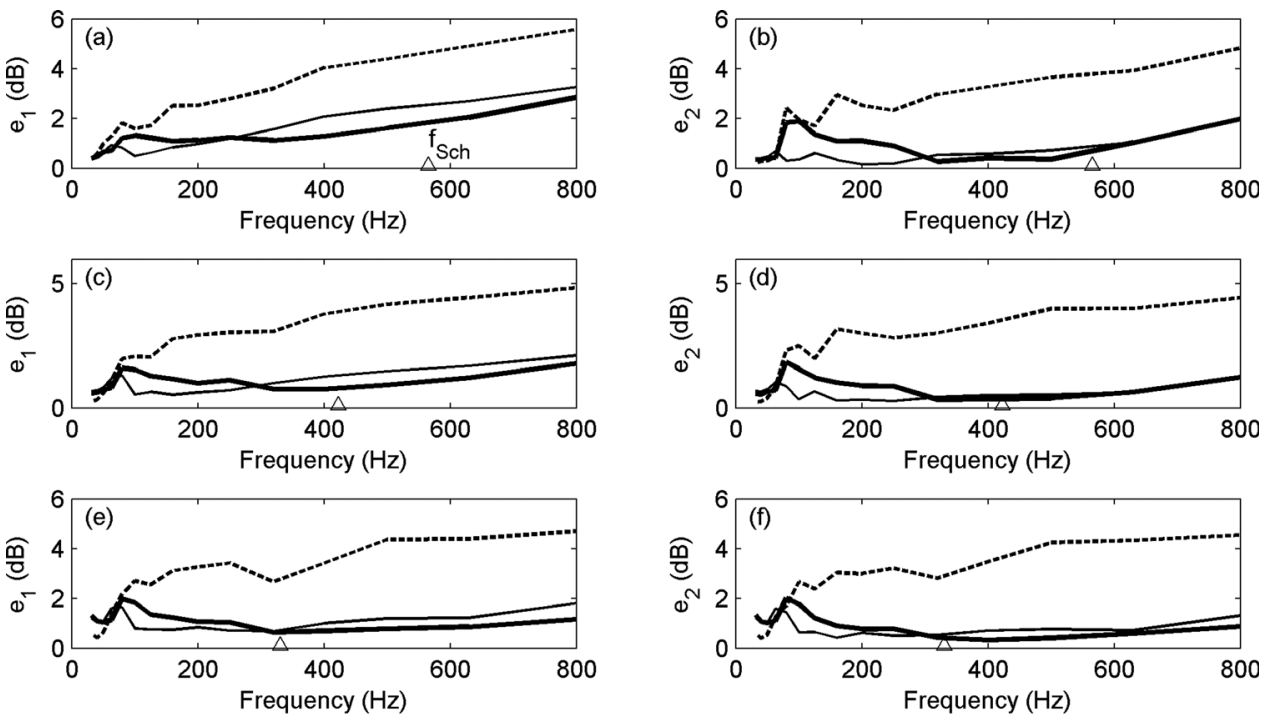

FIG 1. Errors averaged over the receiver positions as a function of frequency in the proportionate room. (a), (c), (e), (g), and (i) $e_{1}$; (b), (d), (f), (h), and (j) $e_{2}$. (a) and (b) $\mathrm{BC} 1$; (c) and (d) BC2; (e) and (f) BC3; (g) and (h) BC4; (i) and (j) BC5.
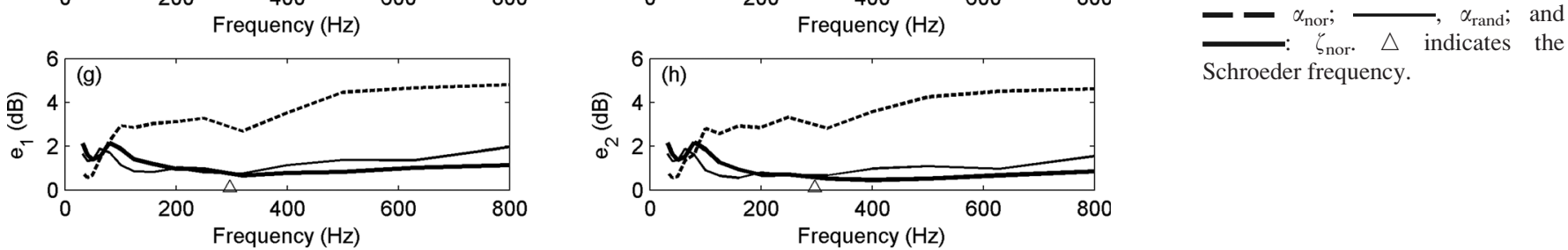
Schroeder frequency.
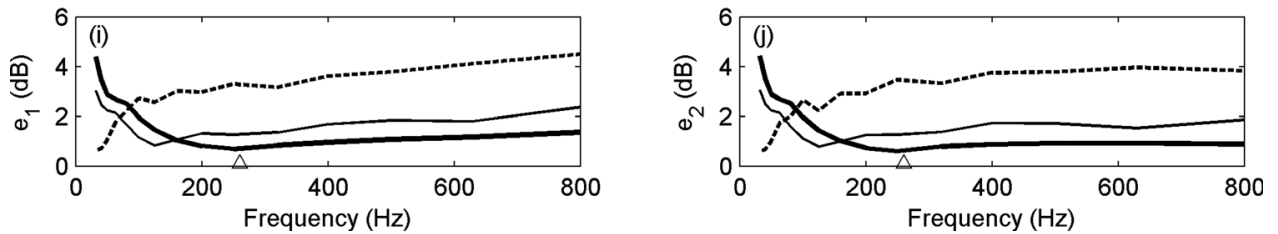

the errors using $\alpha_{\text {field }}$ are only marginally different from the errors using $\alpha_{\text {rand }}$. This is mainly because these two absorption coefficients are very similar as shown in Table I, therefore the errors for $\alpha_{\text {rand }}$, which is more widely used than $\alpha_{\text {field }}$, are only shown in Figs. 1 and 2.

Figure 1 shows the averaged errors over the receiver positions as a function of the frequency for $\alpha_{\text {nor }}, \alpha_{\text {rand }}$, and $\zeta_{\text {nor }}$. For the lowest absorption case, BC1, the $1 / 3$ octave band error is significantly lower than the narrow band error. For $e_{1}$, the random incidence absorption boundary condition shows best results below $200 \mathrm{~Hz}$, whereas the impedance boundary condition yields the best results at higher frequencies. The errors are increased as the frequency increases. For $e_{2}$, the random incidence absorption and impedance boundary conditions yields similar results at frequencies higher than $300 \mathrm{~Hz}$, whereas better agreements are found for the random incidence absorption boundary condition below $300 \mathrm{~Hz}$. The normal incidence absorption coefficient boundary condition yields the worst results.

$\mathrm{BC} 2$ shows similar results to $\mathrm{BC} 1$. However, the errors become lower than those for $\mathrm{BC} 1$, because the simulation errors at the off-resonance frequencies become alleviated for higher absorption cases. ${ }^{3}$ Among the three boundary conditions $\left(\alpha_{\text {nor }}, \alpha_{\text {rand }}\right.$, and $\left.\zeta_{\text {nor }}\right)$, the lowest $e_{1}$ is found for the impedance boundary condition, whereas the lowest $e_{2}$ is observed using the random incidence absorption in Table I. This is mainly ascribed to the fact that the impedance boundary condition slightly underestimates the room response at frequencies below $300 \mathrm{~Hz}$. Beyond the Schroeder frequency of $423 \mathrm{~Hz}$, the simulation accuracy employing the impedance boundary is enhanced and at least comparable to the random incidence absorption boundary condition. Again, the normal incidence absorption coefficient boundary condition yields the worst results.

For $\mathrm{BC} 3$, the lowest $e_{2}$ is found among the tested boundary conditions in Table I. The impedance boundary condition underestimates the room response at frequencies below $300 \mathrm{~Hz}$, but the errors are reduced above the Schroeder frequency. The best boundary condition in terms of $e_{1}$ is the impedance data, whereas a slightly better result is found for the random incidence absorption boundary in terms of $e_{2}$. At frequencies lower than $60 \mathrm{~Hz}$, the normal incidence absorption boundary produces the best results.

For BC4, increased errors are noticed for the random incidence absorption and impedance boundary conditions at low frequencies, whereas the normal incidence absorption boundary condition yields the best result. Above $80 \mathrm{~Hz}$, the errors using the random incidence absorption and impedance boundary conditions tend to be lower than those using the normal incidence absorption coefficient, and the best correspondence is found for the impedance boundary condition above the Schroeder frequency.

For BC5, noticeably amplified errors are found at low frequencies, whereas the high frequency errors are quite 

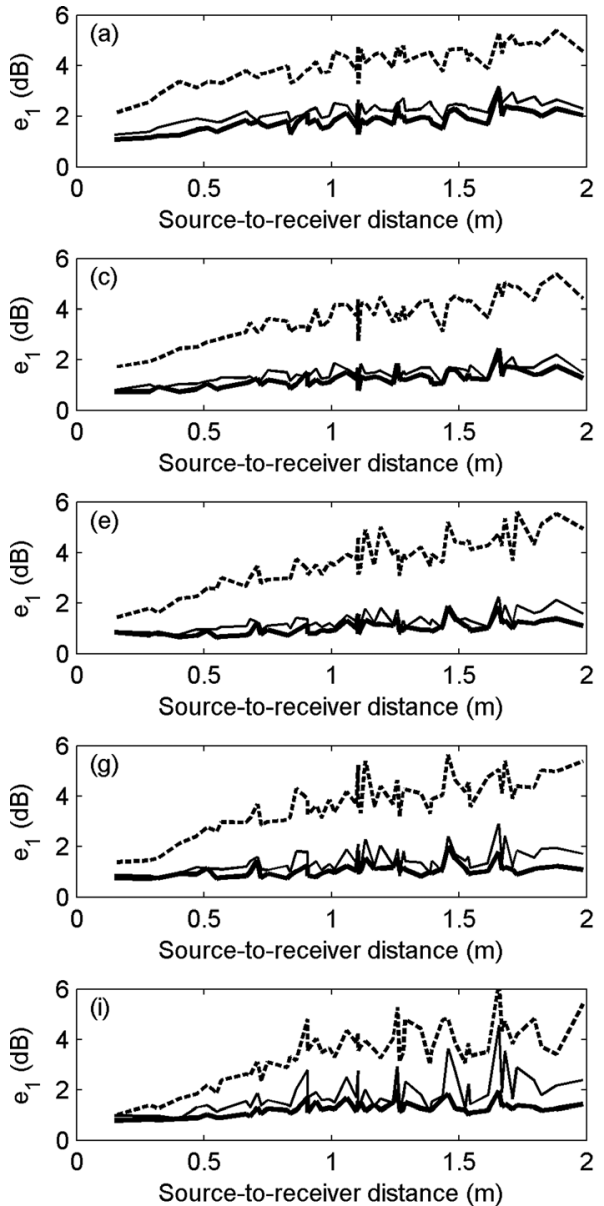
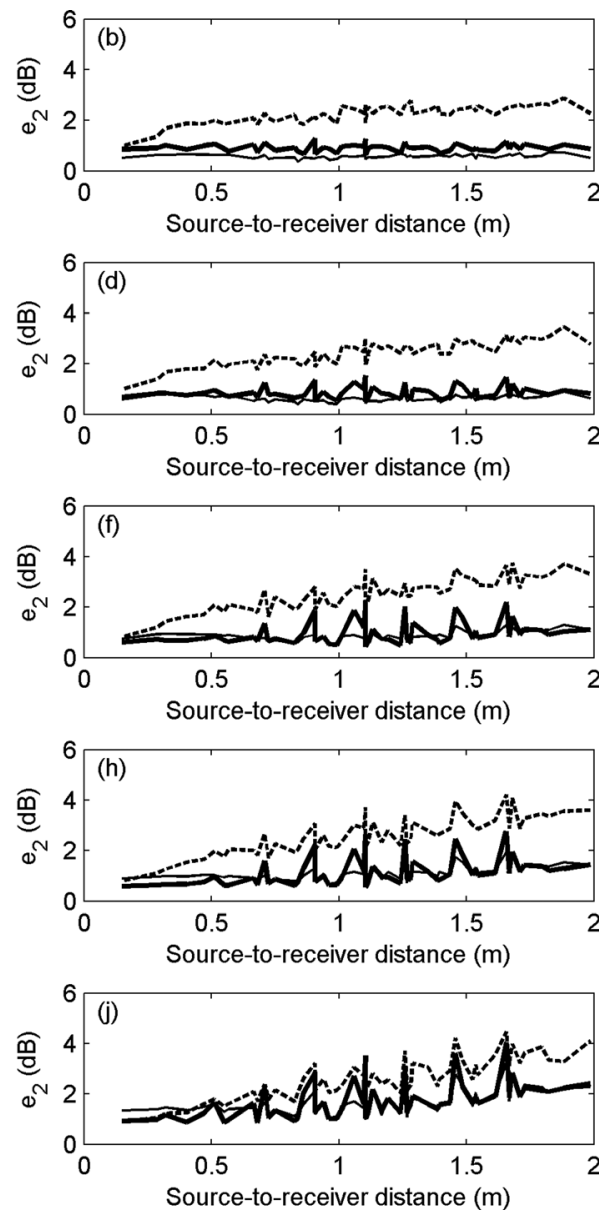

FIG. 2. Errors averaged over the frequency as a function of the source-toreceiver distance in the proportionate room. (a), (c), (e), (g), and (i) $e_{1}$; (b), (d), (f), (h), and (j) $e_{2}$. (a) and (b) $\mathrm{BC} 1$; (c) and (d) $\mathrm{BC} 2$; (e) and (f) BC3; (g) and (h) BC4; (i) and (j) BC5. - $-\alpha_{\text {nor }} ; \longrightarrow, \alpha_{\text {rand }}$; reduced for the impedance and random incidence boundary condition. The normal incidence absorption coefficient boundary condition yields the lowest error at low frequencies. Above $90 \mathrm{~Hz}$, the errors using the random incidence absorption and impedance boundary conditions become lower, and the best correspondences are found for the impedance boundary condition above the Schroeder frequency. Note that $e_{2}$ is larger than $e_{1}$ for the impedance boundary condition because large errors are found at low frequencies.

For the three low absorption cases (BC1-BC3), the random incidence absorption boundary condition best approximates the local reaction boundary condition at low frequencies. Above the Schroeder frequency, the impedance boundary condition agrees best with the boundary element simulations. $e_{2}$ is smaller than $e_{1}$ as can be seen in Table I; this indicates that the errors at the resonance frequencies are likely to be lower than those at the off-resonance frequencies as also pointed out in Refs. 2 and 3. PBTM is inherently more accurate at the resonance frequencies, therefore the calculated $1 / 3$ octave band spectra are more accurate. Generally the normal incidence absorption coefficient leads to the most inaccurate simulations, whereas the random/field incidence absorption and impedance boundary condition represent the locally reacting surfaces better. However, for the first few axial modes below $100 \mathrm{~Hz}$, the normal incidence absorption boundary condition yields a similar agreement to the other boundary conditions; this is not surprising because it is obvious that the sound propagation is one dimensional. Roughly speaking, below the Schroeder frequency, the random incidence absorption boundary condition is better, whereas the accurate results are guaranteed with the impedance boundary condition above the Schroeder frequency. The discrepancies between the random incidence absorption and impedance boundary condition, however, are quite small above the Schroeder frequency. For the very low absorption case, the errors are increased as the frequency increases.

For the high absorption cases, the random incidence absorption and impedance boundary conditions yield reliable results at high frequencies, whereas the normal incidence absorption produces the best results below $100 \mathrm{~Hz}$. This is related to the sphericity error for high absorptions at very low frequencies as Lam and Ingard already pointed out ${ }^{2,27}$ because only the plane-wave reflection coefficient is employed in the PBTM simulations. As Ingard found, the sphericity error is indeed related to the angle of incidence: The sphericity error for oblique angle incidence becomes most significant for the lowest impedance, BC5, which supports the predominance of the incident energy at near normal directions. $^{27}$ This is the reason why the use of the normal incidence absorption coefficient ensures accurate results in this frequency range below $100 \mathrm{~Hz}$. The best PBTM simulation can be obtained by combining the boundary conditions: the normal incidence absorption coefficient below $70 \mathrm{~Hz}$, the random incidence absorption between 70 and $150 \mathrm{~Hz}$, and the impedance beyond $150 \mathrm{~Hz}$. However, this suggested combination is specifically for this test room, and therefore cannot be generalized. Because many room acoustic simulations do not require responses in a very low frequency range, 

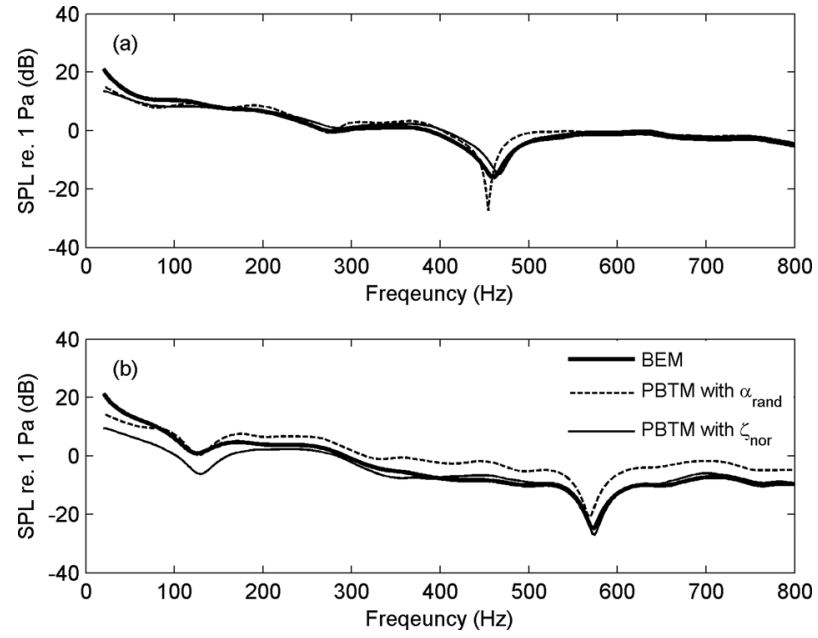

FIG. 3. Simulated transfer functions in the proportionate room. (a) At $(0.75$, $0.2,0.3)$; (b) at $(1.75,0.2,0.3)$.

say below $100 \mathrm{~Hz}$, the random incidence absorption, the field incidence absorption, and impedance boundary conditions are recommended in most cases.

Figure 2 shows the averaged error over the frequency as a function of the source-and-receiver distance. Among the boundary conditions, the normal incidence absorption boundary condition yields the most inaccurate predictions, while the random incidence absorption and impedance boundary conditions produce small errors. The lowest $e_{1}$ is found using the impedance boundary condition. The random incidence absorption boundary condition produces the lowest $e_{2}$ for the three lowest boundary conditions (BC1-BC3), but both impedance and random incidence absorption boundary conditions also produce the best results for $\mathrm{BC} 4$ and BC5 as shown in Table II.

At some specific receiver locations, the errors are amplified. From Figs. 2(b), 2(d), 2(f), and 2(h), it is observed that the $1 / 3$ octave band error using the random incidence absorption boundary condition is less influenced by the receiver position than using the impedance boundary condition. From Fig. 2(j), large errors using the impedance boundary condition are found for specific receiver locations at $(0.15,1.0$, $0.3),(0.15,1.2,0.3),(1.35,0.2,0.3),(1.55,0.2,0.3)$, and $(1.75,0.2,0.3)$. These receivers have a common aspect that at least one of the first order reflections has a large incidence angle. The largest incidence angles of the first order reflections for the listed five receivers are $73.7^{\circ}, 77.0^{\circ}, 76.2^{\circ}$, $79.1^{\circ}$, and $78.8^{\circ}$, respectively. For such grazing incidence, the plane wave reflection coefficient by Eq. (7) is known to be incorrect and therefore yields large errors by using the impedance boundary condition. ${ }^{23,28}$ To avoid such errors using the impedance boundary condition that depend on the source and receiver location, one may use the random incidence absorption boundary condition.

Figure 3 compares transfer functions at two receiver locations for BC5: one of the problematic receivers at (1.75, $0.2,0.3)$ and a receiver having low errors at $(0.75,0.2,0.3)$. The $1 / 3$ octave band errors are 4.0 and $1.6 \mathrm{~dB}$, respectively. The main differences are found at low frequencies: Larger errors are found below $300 \mathrm{~Hz}$ for the problematic receiver in Fig. 3(b), whereas the other receiver exhibits much smaller errors in the same frequency range in Fig. 3(a). Such low frequency errors amplify $e_{2}$, although the high frequency errors are quite acceptable as can be seen in Figs. 1 and 3. Because $e_{1}$ has a uniform weighting over the frequency of interest, $e_{1}$ is always lowest with the impedance boundary condition that yields accurate high frequency responses. Figure 3 also reveals that the impedance boundary condition is actually superior to the random incidence absorption boundary condition in terms of the identification of the locations and levels of the troughs.

Additionally, the reverberation times for the investigated boundary conditions are calculated. From the calculated transfer functions, impulse responses are computed via the inverse Fourier transformation. $T_{20}$ is calculated in octave bands for the impedance and random incidence boundary conditions by the backward integration method ${ }^{29}$ and listed in Table V. Compared with the reference cases $\left(\mathrm{BEM}_{\zeta \text { nor }}\right)$, the differences are fairly small. The differences for the random incidence boundary conditions are smaller at low frequencies, but the differences for the impedance boundary conditions become smaller in the $500 \mathrm{~Hz}$ octave band. The reverberation time results also support that the impedance boundary condition provides the best results at high frequencies, whereas the random incidence absorption boundary condition is the best at low frequencies.

\section{B. Non-uniform absorption in the proportionate room}

A non-uniform distribution of absorption was simulated. A typical lecture room has a large amount of absorption treated on the ceiling, but has acoustically reflective walls. Therefore the impedances for the ceiling, floor, and all the side walls were chosen as 5.9, 18, and 38, respectively, which correspond to the random absorption coefficients for the ceiling, floor, and side walls of $0.66,0.32$, and 0.17 , respectively. The average absorption coefficients in terms of

TABLE V. Reverberation times $\left(T_{20}\right)$ in octave bands for different boundary conditions. Differences larger than $10 \%$ is indicated in bold.

\begin{tabular}{|c|c|c|c|c|c|c|c|c|c|c|c|c|}
\hline \multirow{2}{*}{$\frac{f_{\mathrm{c}}}{\mathrm{BC}}$} & \multicolumn{3}{|c|}{$63 \mathrm{~Hz}$} & \multicolumn{3}{|c|}{$125 \mathrm{~Hz}$} & \multicolumn{3}{|c|}{$250 \mathrm{~Hz}$} & \multicolumn{3}{|c|}{$500 \mathrm{~Hz}$} \\
\hline & $\mathrm{BEM} \zeta_{\text {nor }}$ & PBTM $\zeta_{\text {nor }}$ & $\operatorname{PBTM} \alpha_{\text {rand }}$ & $\mathrm{BEM} \zeta_{\text {nor }}$ & $\operatorname{PBTM} \zeta_{\text {nor }}$ & $\operatorname{PBTM} \alpha_{\text {rand }}$ & $\mathrm{BEM} \zeta_{\text {nor }}$ & PBTM $\zeta_{\text {nor }}$ & $\operatorname{PBTM} \alpha_{\text {rand }}$ & $\mathrm{BEM} \zeta_{\text {nor }}$ & PBTM $\zeta_{\text {nor }}$ & $\operatorname{PBTM} \alpha_{\text {rand }}$ \\
\hline $\mathrm{BC} 1$ & 0.25 & 0.23 & 0.26 & 0.27 & 0.20 & 0.27 & 0.24 & 0.19 & 0.24 & 0.18 & 0.19 & 0.21 \\
\hline $\mathrm{BC} 2$ & 0.23 & 0.24 & 0.24 & 0.19 & 0.16 & 0.20 & 0.14 & 0.11 & 0.16 & 0.11 & 0.10 & 0.12 \\
\hline $\mathrm{BC} 3$ & 0.24 & 0.26 & 0.25 & 0.15 & 0.13 & 0.15 & 0.09 & 0.07 & 0.10 & 0.07 & 0.06 & 0.07 \\
\hline $\mathrm{BC} 4$ & 0.25 & 0.26 & 0.26 & 0.13 & 0.12 & 0.14 & 0.07 & 0.06 & 0.08 & 0.04 & 0.04 & 0.06 \\
\hline BC5 & 0.26 & 0.26 & 0.26 & 0.12 & 0.12 & 0.12 & 0.06 & 0.06 & 0.06 & 0.03 & 0.03 & 0.04 \\
\hline
\end{tabular}


the normal and random incidence absorption were calculated as 0.21 and 0.31 , which is similar to BC2 in Table I. The Schroeder frequency of the room is $414 \mathrm{~Hz}$. Figure 4 shows the error as a function of the center frequency of the $1 / 3$ octave bands. Similar error trends are found: The impedance boundary condition yields the lowest $e_{1}$ except for the very low frequency bands, whereas the random incidence absorption coefficient boundary condition guarantees the lowest $e_{2}$ except for the $800 \mathrm{~Hz}$ band.

Compared with $\mathrm{BC} 2$, which has a similar mean absorption coefficient, the averaged errors are increased for the non-uniform absorption configuration; the lowest $e_{1}$ for the impedance boundary condition is increased by $1 \mathrm{~dB}$ and the lowest $e_{2}$ for the random incidence absorption boundary condition by $0.6 \mathrm{~dB}$. Note that this non-uniform distribution result was already published in the previous work ${ }^{3}$ but only up to the Schroeder frequency. In this study, the transfer functions were further simulated up to $1000 \mathrm{~Hz}$, and the error trends were analyzed as a function of frequency. The reason for the increased error was already discussed in the previous paper that the phased beam tracing used cannot account for wave phenomena due to the discontinuity in the boundary condition, i.e., wave diffraction and scattering evoked by the discontinuity in the boundary condition. ${ }^{3}$

\section{Neglect of phase shift on reflection}

In actual situations, true pressure reflection coefficients and surface impedances are complex-valued, and therefore the reflection phase should not be ignored. In this sense, the impedance boundary condition has a definite advantage that it can take account of phase shift on reflection. However, in most cases, such reflection phase is difficult to estimate, therefore real-valued boundary conditions are predominantly used in practice. In this section, two scenarios are studied on how the real-valued approximate boundary conditions influ-
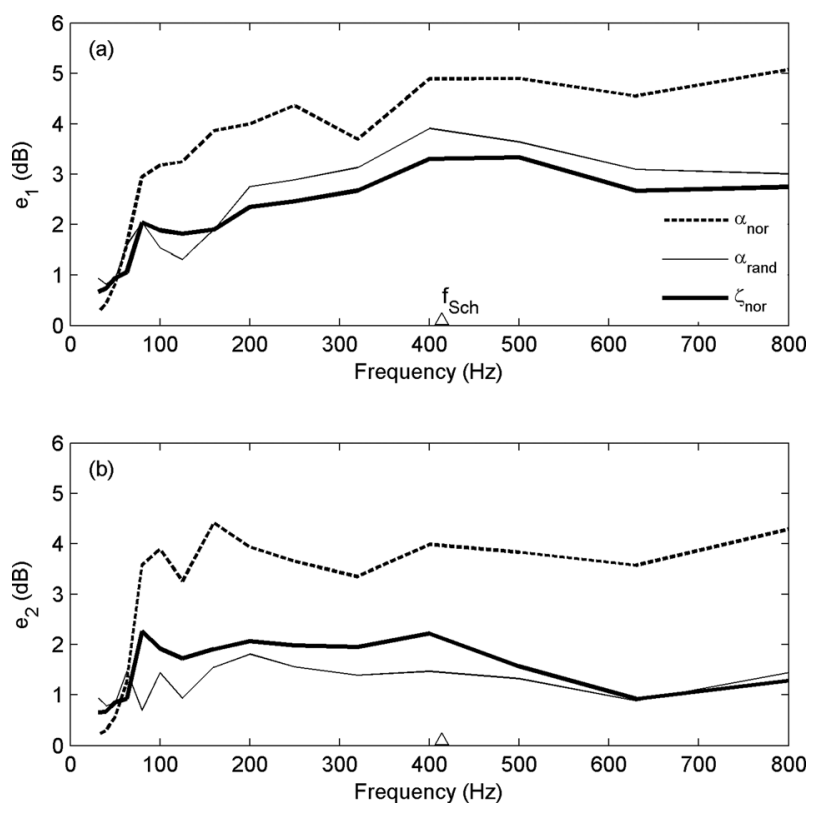

FIG. 4. Errors averaged over the receiver positions as a function of frequency in the proportionate room for a non-uniform absorption configuration. (a) $e_{1}$; (b) $e_{2} . \triangle$ indicates the Schroeder frequency. ence simulation results. Assume a uniformly distributed absorption in the proportionate room. First, the magnitude of the specific surface impedance is assumed to be constant as 20 , and the phase angle of the specific impedance, $\mu$ as arc$\tan \left(\operatorname{Im}\left(\zeta_{\text {nor }}\right) / \operatorname{Re}\left(\zeta_{\text {nor }}\right)\right)$, changes from $0^{\circ}$ to $60^{\circ}$. Note that the zero phase angle case corresponds to $\mathrm{BC} 2$ in Table I. The other three phase angles are chosen to be $30^{\circ}, 45^{\circ}$, and $60^{\circ}$. In Table II, the complex boundary conditions and corresponding absorption coefficients are listed. As the phase angle increases, the equivalent absorption decreases. Therefore it is likely that the PBTM simulation with BC2 differs from the other simulations employing the complex-valued boundary conditions. Figure 5 shows a comparison of the boundary element simulations with three phase angles, $0^{\circ}$, $30^{\circ}$, and $60^{\circ}$ at the closest receiver location at $(0.15,0.2$, $0.3)$. The frequency shifts of the peaks and troughs and their magnitude changes are observed due to the phase shift on reflection.

The PBTM simulations adopting the real-valued impedance (BC2) and complex-valued impedance data (C-BC1 to $\mathrm{C}-\mathrm{BC} 3)$ are compared with the boundary element simulations with the three complex-valued boundary conditions to estimate the additionally increased error due to the neglect of the reflection phase. If the rooms are simulated by PBTM with the correct boundary condition, the maximum $e_{1}$ and $e_{2}$ are 2 and $1.2 \mathrm{~dB}$, respectively, for $\mathrm{C}-\mathrm{BC} 3$ as shown in Table III. If the approximate real-valued impedance boundary condition is assigned, the simulation errors are increased up to 4.4 and $3.5 \mathrm{~dB}$, respectively. Therefore the maximum increase due to the neglect of the phase angle in the impedance boundary condition is about $2.4 \mathrm{~dB}$ for $e_{1}$ and $2.3 \mathrm{~dB}$ for $e_{2}$. These significant increases in both $e_{1}$ and $e_{2}$ indicate that the simulation accuracy is degraded at both resonance frequencies and off-resonance frequencies: The frequency shifts and the magnitude changes in the peaks mainly increase $e_{1}$, whereas only the magnitude changes in the peaks increase $e_{2}$. Another interesting finding is that as the phase angle of the impedance increases, the accuracy of the PBTM simulations even with the correct boundary conditions become degraded; $e_{1}$ and $e_{2}$ are increased by 0.8 and $0.3 \mathrm{~dB}$, respectively, for the phase angle of $60^{\circ}$. However, such a real-valued impedance approximation is not highly

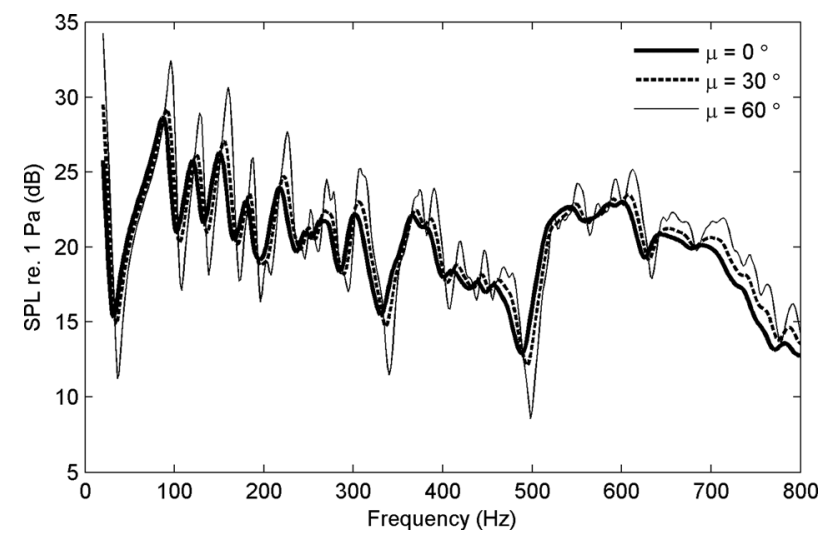

FIG. 5. Simulated transfer functions by the boundary element method at $(0.15,0.2,0.3)$ using different boundary conditions. 
likely because one can obtain the correct phase angle information when measuring the surface impedance by the duct method.

A more realistic scenario is to compare various surface impedance values that are equivalent to an identical absorption coefficient. This happens when one knows an approximate absorption coefficient of the surface of interest and tries to estimate the surface impedance from the approximate absorption coefficients without measurements. There are an infinite number of combinations of the real and imaginary part of the impedance for a fixed absorption coefficient. Assuming the zero phase angle of the surface impedance, the surface impedance of 20 corresponds to a random incidence absorption coefficient of 0.3 . Table III lists three other complex-valued surface impedances having phase angles of $30^{\circ}, 45^{\circ}$, and $60^{\circ}$, equivalent to the absorption coefficient of 0.3 . Boundary element simulations with the three correct complex-valued impedance boundary conditions are compared with the PBTM simulations using the random incidence absorption coefficient of 0.3 and the correct complex-valued impedance boundary conditions.

Using the random incidence absorption boundary condition, the phase shift on reflection is neglected by Eq. (8), therefore the lowest error is expected for $\mathrm{BC} 2$ for which the correct reflection phase is also zero. In terms of $e_{1}$, the approximate random incidence absorption coefficient degrades the simulation accuracy by $3 \mathrm{~dB}$ for the phase angle of $60^{\circ}$, $2.1 \mathrm{~dB}$ for $45^{\circ}, 1.4 \mathrm{~dB}$ for $30^{\circ}$, and $0.2 \mathrm{~dB}$ for $0^{\circ}$. In terms of $e_{2}$, the increase in $e_{2}$ can be as large as $0.9 \mathrm{~dB}$, which is relatively small compared with the increase in $e_{1}$. With the neglect of the reflection phase, the PBTM simulation accuracy is degraded to some degree; the larger the phase angle of the impedance, the larger the additional error due to the neglect of the reflection phase shift.

\section{Uniform absorption in the disproportionate room}

Again, the five uniform boundary conditions are assigned on the boundary walls in the disproportionate room. It has been found that the more disproportionate the room, the larger the errors. ${ }^{3}$ In Table IV, average errors over the frequency range and the receiver positions are listed. In terms of the narrow band error $e_{1}$, the impedance boundary condition always yields the best results. However, the errors are quite increased compared with the proportional room case, e.g., for $\mathrm{BC} 5$, the lowest $e_{1}$ and $e_{2}$ are increased by $1.2 \mathrm{~dB}(1.2 \mathrm{~dB} \rightarrow 2.4 \mathrm{~dB})$ and $2.5 \mathrm{~dB}(1.7 \mathrm{~dB} \rightarrow 4.2 \mathrm{~dB})$, respectively. It turns out that the random incidence absorption boundary condition consistently yields the best results in terms of $e_{2}$, but the differences in $e_{2}$ between the random incidence absorption and the impedance boundary condition are all less than $0.3 \mathrm{~dB}$ as shown in Table IV.

These errors are plotted as a function of the frequency in Fig. 6. The random incidence absorption boundary condition is superior at frequencies below $200 \mathrm{~Hz}$, whereas the impedance boundary condition agrees best with the boundary
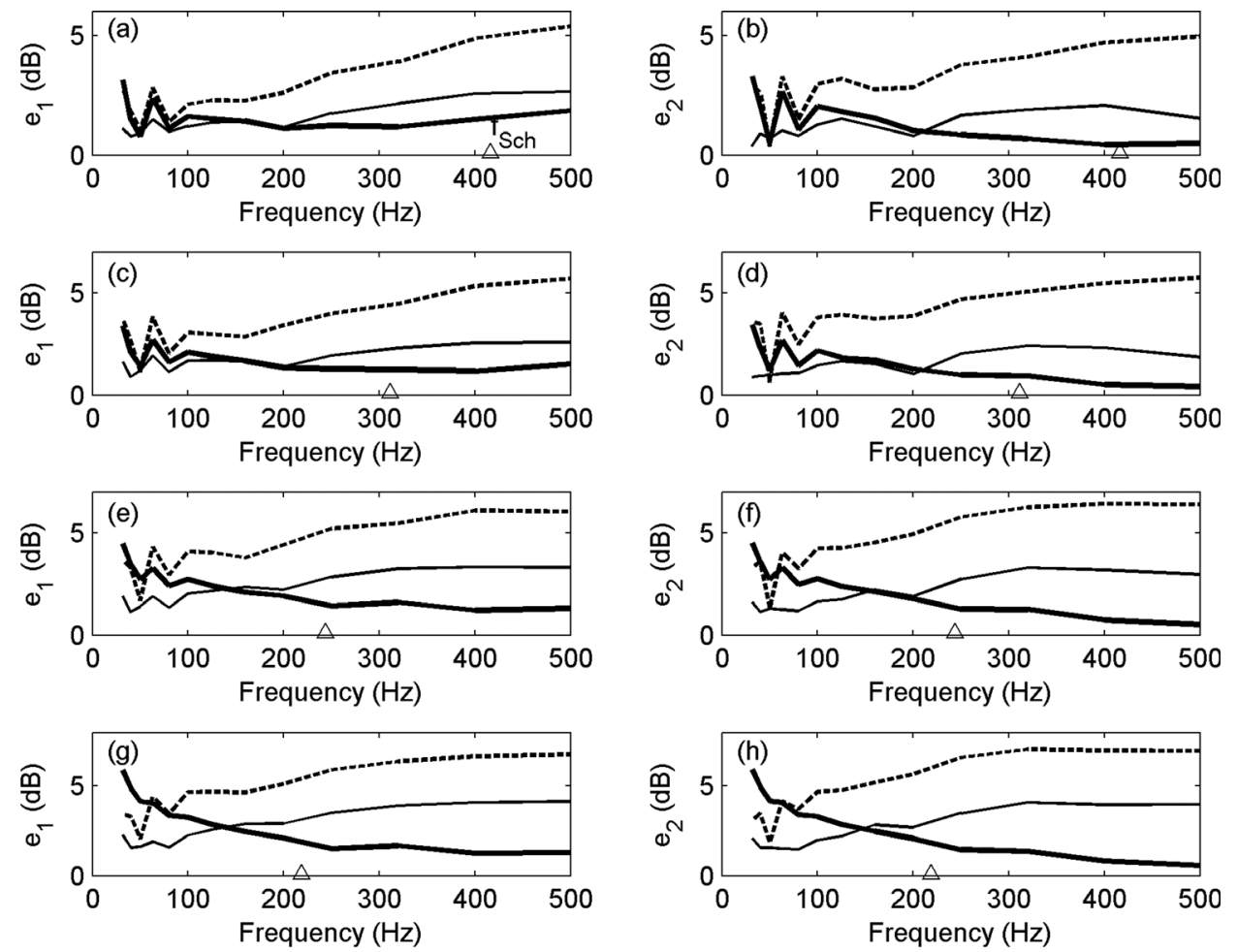

FIG. 6. Errors averaged over the receiver positions as a function of frequency in the disproportionate room. (a), (c), (e), (g), and (i) $e_{1}$; (b), (d), (f), (h), and (j) $e_{2}$. (a) and (b) $\mathrm{BC} 1$; (c) and (d) $\mathrm{BC} 2$; (e) and (f) $\mathrm{BC} 3$; (g) and (h) BC4; (i) and (j) BC5. -,$\alpha_{\text {nor }} ; \underset{\text { indicates }}{-}, \alpha_{\text {rand }}$; Schroeder frequency.
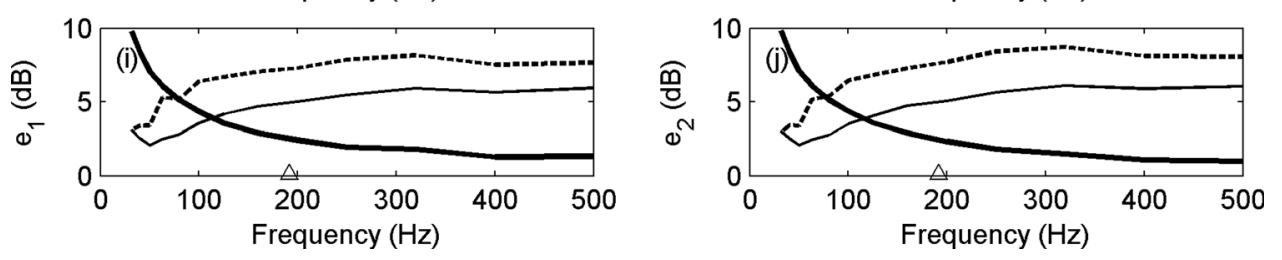
element simulations above $200 \mathrm{~Hz}$. Note increased errors in Fig. 4(j) at frequencies lower than $100 \mathrm{~Hz}$ due to the spherical error, but the error employing the impedance boundary condition decreases significantly as the frequency increases. The random incidence absorption boundary condition consistently produces the lowest error even at low frequency for low impedances than the normal incidence absorption coefficients in terms of both $e_{1}$ and $e_{2}$.

\section{SUMMARY}

Generally speaking, the impedance, random incidence absorption, and field incidence absorption boundary conditions yield reasonably accurate results. The impedance boundary condition together with the plane wave reflection modeling is found to be acceptable. If narrow band spectra are to be calculated, the impedance boundary condition is strongly recommended. Even for 1/3 octave band spectra, the error with the impedance boundary condition is increased by only maximum $0.3 \mathrm{~dB}$ compared with the random incidence boundary condition. At high frequencies, particularly above the Schroeder cutoff frequency, the impedance boundary condition always produces the best results. On the other hand, for low impedance at very low frequencies, the sphericity error dominates, therefore the impedance boundary condition with the plane-wave reflection modeling becomes problematic. The impedance boundary condition exhibits large errors at receivers with nearly grazing incidence angles for the first order reflection. In other words, the simulation errors depend more on the source and receiver location with the impedance boundary condition.

The random incidence and field incidence absorption boundary conditions are also quite acceptable if the phase shift on reflection is close to zero. If $1 / 3$ octave band spectra are to be calculated, these absorptions boundary conditions are likely to be good boundary conditions. As the reflection phase change increases, these approximate absorption boundary conditions cannot predict the sound field well, and the error can be additionally increased by up to $3 \mathrm{~dB}$. These boundary conditions are more robust regardless of the source and receiver location but again only for nearly zero phase shift on reflection. They are relatively easy to estimate, but great care must be taken to distinguish these quantities from Sabine absorption coefficients.

The normal incidence absorption is least accurate in general. This type of boundary condition is useful in a very limited low frequency range, say, up to a few axial modes. For acoustically soft materials, this quantity can be used at the very low frequencies. The normal incidence boundary condition generally increases the simulation error by around $2 \mathrm{~dB}$ relative to the other boundary conditions.

\section{CONCLUSIONS}

This study investigates absorption and impedance boundary conditions for a phased beam tracing model. PBTM simulations employing normal incidence absorption, random incidence absorption, field incidence absorption, and impedance boundary conditions are compared with boundary element solutions in two rectangular rooms under the assumption of locally reacting boundaries. A wide range of impedance and absorption boundary conditions are tested with uniform and non-uniform absorption configurations. Neglect of the phase shift on reflection is also studied.

The impedance boundary condition is found to yield the best results at higher frequencies above the Schroeder frequency with a few exceptions, whereas the random/field incidence absorption coefficient boundary conditions are proven to be robust, in particular at low frequencies. The normal incidence boundary condition generally produces larger errors, and this type of boundary condition is only acceptable for the first few axial room modes and/or for the boundaries having low impedance or high absorption. A non-uniform boundary configuration shows similar error trends for the tested boundary conditions, but increases the errors by about $1 \mathrm{~dB}$ due to wave phenomena evoked by the discontinuous acoustic properties at the boundary. By using the plane wave reflection coefficients computed from the impedance boundary condition, the simulation errors can be amplified at frequencies below $100 \mathrm{~Hz}$ for low impedance/ high absorption. This sphericity error, however, occurs only at very low frequencies, therefore in most cases, the impedance boundary condition with plane-wave reflection modeling is a reliable boundary condition. At receiver locations having nearly grazing first-order reflection components, the errors are amplified by the plane-wave reflection modeling, whereas the random/field incidence boundary conditions are less affected by the source and receiver location, which is a positive aspect. For the disproportionate room, the errors are increased, but the error trend of the boundary conditions investigated is similar to the proportionate room case. For a correct phase angle of $60^{\circ}$, the simulation error can be increased by $2.4 \mathrm{~dB}$ for the wrong estimation of the phase angle of the impedance and by $3 \mathrm{~dB}$ when using the random incidence absorption boundary condition. In many cases, it is difficult to characterize the reflection phase, therefore one always bears such a fairly large amount of the increased error in mind when simulating sound fields with approximate absorption/impedance boundary conditions.

${ }^{1}$ J. S. Suh and P. A. Nelson, "Measurement of transient response of rooms and comparison with geometrical acoustic models," J. Acoust. Soc. Am. 105, 2304-2317 (1999).

${ }^{2}$ Y. W. Lam, "Issues for computer modelling of room acoustics in nonconcert hall settings," Acoust. Sci. Tech. 26, 145-155 (2005).

${ }^{3}$ C.-H. Jeong and J.-G. Ih, "Effects of source and receiver locations in predicting room transfer functions by a phased beam tracing method," J. Acoust. Soc. Am. 131, 3864-3875 (2012).

${ }^{4}$ A. Wareing and M. Hodgson, "Beam-tracing model for predicting sound field in rooms with multilayer bounding surfaces," J. Acoust. Soc. Am. 118, 2321-2331 (2005).

${ }^{5} \mathrm{M}$. Aretz and M. Vorländer, "Efficient modelling of absorbing boundaries in room acoustic FE simulations," Acta Acust. Acust. 96, 1042-1050 (2010).

${ }^{6}$ Z. Maekawa, J. H. Rindel, and P. Lord, Environmental and Architectural Acoustics, 2nd ed. (Spon Press, London, 2011), Chap. 4.

${ }^{7}$ ISO 354:2003: Acoustics-Measurement of Sound Absorption in a Reverberation Room (International Organization for Standardization, Geneva, 2003).

${ }^{8} \mathrm{C}$. W. Kosten, "International comparison measurement in the reverberation room," Acustica 10, 400-411 (1960). 
${ }^{9} \mathrm{M}$. Vercammen, "Improving the accuracy of sound absorption measurement according to ISO 354," Proceedings of the International Symposium on Room Acoustics, Melbourne, Australia (2010).

${ }^{10}$ H. Kuttruff, Room Acoustics, 4th ed. (Spon Press, London, 2000), Chap. 2.5 .

${ }^{11}$ ASTM C423:2009: Standard Test Method for Sound Absorption and Sound Absorption Coefficients by the Reverberation Room Method (American Society for Testing and Materials International, West Conshohocken, PA, 2009).

${ }^{12}$ K. Attenborough, "Acoustical characteristics of porous materials," Phys. Rep. 82, 179-227 (1982).

${ }^{13} \mathrm{C}$. Klein and A. Cops, "Angle dependence of impedance of a porous layer," Acustica 44, 258-264 (1980).

${ }^{14}$ D. B. Bliss and S. E. Burke, "Experimental investigation of the bulk reaction boundary condition," J. Acoust. Soc. Am. 71, 546-551 (1982).

${ }^{15}$ D. J. Sides and K. J. Mulholland, "The variation of normal layer impedance with angle of incidence," J. Sound Vib. 14, 139-142 (1971).

${ }^{16}$ E. A. G. Shaw, "The acoustic wave guide. II. Some specific normal acoustic impedance measurements of typical porous surfaces with respect of normally and obliquely incident waves," J. Acoust. Soc. Am. 25, 231-235 (1953).

${ }^{17}$ C.-H. Jeong, "Guideline for adopting the local reaction assumption for porous absorbers in terms of random incidence absorption coefficients," Acta Acust. Acust. 97, 779-790 (2011).

${ }^{18} \mathrm{M}$. Hodgson, and A. Wareing, "Comparisons of predicted steady-state levels in rooms with extended- and local-reaction bounding surfaces," J. Sound Vib. 309, 167-177 (2008).
${ }^{19}$ M. M. Louden, "Dimension ratios of rectangular rooms with good distribution of eigentones," Acustica 24, 101-104 (1971).

${ }^{20} \mathrm{~T}$. Lewers, "A combined beam tracing and radiant exchange computermodel of room acoustics," Appl. Acoust. 38, 161-178 (1993).

${ }^{21}$ P. M. Morse and K. U. Ingard, Theoretical Acoustics (McGraw-Hill, New York, 1968), p. 319.

${ }^{22}$ C.-H. Jeong, J.-G. Ih, and J. H. Rindel, "An approximate treatment of reflection coefficient in the phased beam tracing method for the simulation of enclosed sound fields at medium frequencies," Appl. Acoust. 69, 601-613 (2007).

${ }^{23}$ J. H. Rindel, "Modeling the angle-dependent pressure reflection factor," Appl. Acoust. 38, 223-234 (1993).

${ }^{24}$ T. Funkhouser, N. Tsingos, I. Carlbom, G. Elko, M. Sondhi, J. E. West, G. Pingali, P. Min, and A. Ngan, "A beam tracing method for interactive architectural acoustics," J. Acoust. Soc. Am. 115, 739-756 (2004).

${ }^{25}$ I. A. Drumm, and Y. W. Lam, "The adaptive beam-tracing algorithm," J. Acoust. Soc. Am. 107, 1405-1412 (2000).

${ }^{26}$ M. Vorlander, Auralization: Fundamentals of Acoustics, Modelling, Simulation, Algorithms and Acoustic Virtual Reality (Springer-Verlag, Berlin, 2008), pp. 155-156.

${ }^{27} \mathrm{U}$. Ingard, "On the reflection of a spherical sound wave from an infinite plane,” J. Acoust. Soc. Am. 23, 329-335 (1951).

${ }^{28}$ S.-I. Thomasson, "Theory and experiments on the sound absorption as function of the area," Report TRITA-TAK8201, KTH, Stockholm, Sweden (1982).

${ }^{29}$ M. R. Schroeder, "New method of measuring reverberation time," J. Acoust. Soc. Am. 37, 409-412 (1965). 